\title{
Supersymmetric quantum mechanics with nonlocal potentials
}

\author{
Je-Young Choi* \\ Department of Basic Sciences \\ Youngdong University \\ Youngdong, Chungbuk 370-800, KOREA \\ Seok-In Hong ${ }^{\dagger}$ \\ Department of Science Education \\ Inchon National University of Education \\ Inchon 407-753, KOREA
}

(October 3, 2018)

We consider supersymmetric quantum mechanical models with both local and nonlocal potentials. We present a nonlocal deformation of exactly solvable local models. Its energy eigenfunctions and eigenvalues are determined exactly. We observe that both our model Hamiltonian and its supersymmetric partner may have normalizable zero-energy ground states, in contrast to local models with nonperiodic or periodic potentials.

\section{INTRODUCTION}

Nonlocal potentials occur in the single particle descriptions of many-body systems such as the Hartree-Fock approximation [1] and have intrinsic applications in atomic and molecular physics [2], solid-state physics 3, and nuclear physics [- $[$. Nonlocal models are also used in studying statistical properties of extended objects such as polymers and proteins [5].

In this paper we are interested in exactly solvable quantum mechanical models with nonlocal potentials. We resort here to supersymmetric (SUSY) quantum mechanics [6,7] which has been extensively studied recently. SUSY has been applied to exact solvability [8] in connection with shape invariance [9], inverse scattering [10], supersymmetry-inspired WKB method [11], and surface critical phenomena of inhomogeneous Ising models $[12$. It is also generalized to higher dimensions [13, $N$-body problems 14, and dynamics 15 .

In SUSY quantum mechanics, a given Hamiltonian and its SUSY partner have identical spectra except the zeroenergy ground state. In ordinary cases, either one or neither of the partner Hamiltonians has a zero-energy ground state, in which case SUSY is unbroken or broken, respectively. Dunne et al. [16] considered SUSY quantum mechanical models with periodic potentials to find that it is possible for both SUSY partners to have zero-energy ground states. However their ground states are not normalizable because they are Bloch states. In the present paper we consider SUSY quantum mechanical models with both local and nonlocal potentials. We present a simple nonlocal deformation of exactly solvable local models. We find the energy eigenfunctions and eigenvalues exactly and observe that both supersymmetric partners may have, in some parameter range, normalizable zero-energy ground states, which are in contrast to local models with nonperiodic or periodic potentials.

\section{NONLOCAL POTENTIALS AND SUPERSYMMETRY}

The time-independent Schrödinger equation with both local and nonlocal potentials in one dimension is, in the position representation,

$$
\begin{aligned}
-\frac{\hbar^{2}}{2 m} \frac{d^{2}}{d x^{2}} & \psi(x)+V_{-}(x) \psi(x) \\
& \quad+\int_{-\infty}^{+\infty} d y v_{-}(x, y) \psi(y)=E_{-} \psi(x) .
\end{aligned}
$$

The invariance under time reversal and the conservation of the probability require that $V_{-}(x)$ and $v_{-}(x, y)$ are real and that $v_{-}(x, y)$ is symmetric in $x$ and $y$. In the Hartree-Fock approximation the nonlocal potential (or the proper self-energy) is determined self-consistently from the given external local potential and two-body interactions [1]. But in this paper, we assume that the potentials are given in the beginning.

There are trivial choices of potentials which are exactly solvable. Suppose that $\psi_{n}(x)$ are the real normalized eigenstates of a local-potential problem

$$
-\frac{\hbar^{2}}{2 m} \frac{d^{2}}{d x^{2}} \psi_{n}(x)+V_{-}(x) \psi_{n}(x)=E_{n}^{(0)} \psi_{n}(x)
$$

with $n$ the number of nodes of $\psi_{n}(x)$. Then a trivial choice of the nonlocal potential $v_{-}(x, y)$ is of the form

$$
v_{-}(x, y)=\sum_{n} \epsilon_{n} \psi_{n}(x) \psi_{n}(y)
$$


with $\epsilon_{n}$ real numbers. Then $\psi_{n}(x)$ is the eigenfunction of Eq. (11) with energy eigenvalue $E_{n}=E_{n}^{(0)}+\epsilon_{n}$. Hence we see that even though $E_{n}^{(0)}<E_{n+1}^{(0)}$ in local models, $E_{n}<E_{n+1}$ may not hold in models with nonlocal potentials. We may also consider a momentum dependent potential $v(\hat{p})$ 18. In the position representation, it may be alternatively represented as a nonlocal potential

$$
v_{-}(x, y)=\frac{1}{2 \pi \hbar} \int_{-\infty}^{\infty} d p \mathrm{e}^{i p(x-y) / \hbar} v(p),
$$

which depends only on the difference $x-y$. If we further restrict the local potential $V_{-}(x)$ to be harmonic, then in the momentum representation, we have

$$
-\frac{1}{2} m \omega^{2} \hbar^{2} \frac{d^{2}}{d p^{2}} \psi(p)+\left(\frac{p^{2}}{2 m}+v(p)\right) \psi(p)=E \psi(p),
$$

which is of the same form as the local-potential problem (2) in the position representation, so that we may exploit the solvable models of local potentials. In addition, nonlocal separable potentials also lead to solvable models [17]. In the following we will exclude these cases.

Sometimes it is convenient to write potentials in operator form as

$$
\begin{aligned}
& \hat{V}_{-}=\int_{-\infty}^{\infty} d x|x\rangle V_{-}(x)\langle x|, \\
& \hat{v}_{-}=\int_{-\infty}^{\infty} d x \int_{-\infty}^{\infty} d y|x\rangle v_{-}(x, y)\langle y| .
\end{aligned}
$$

Then the Hamiltonian is

$$
\hat{H}_{-}=\frac{\hat{p}^{2}}{2 m}+\hat{V}_{-}+\hat{v}_{-} .
$$

As in SUSY quantum mechanics with local potentials [7], we assume that the Hamiltonian $\hat{H}_{-}$can be factorized in the form $\hat{H}_{-}=\hat{A}^{\dagger} \hat{A}$. Its SUSY partner Hamiltonian $\hat{H}_{+}$ is then given by

$$
\hat{H}_{+}=\hat{A} \hat{A}^{\dagger}=\frac{\hat{p}^{2}}{2 m}+\hat{V}_{+}+\hat{v}_{+} .
$$

First-order differential operators $\hat{A}^{\dagger}$ and $\hat{A}$ are defined by

$$
\begin{gathered}
\hat{A}^{\dagger}=-\frac{i \hat{p}}{\sqrt{2 m}}+\hat{W}+\hat{w}, \\
\hat{A}=\frac{i \hat{p}}{\sqrt{2 m}}+\hat{W}+\hat{w},
\end{gathered}
$$

where $\hat{W}$ and $\hat{w}$ are defined by Eq. (6) with $V_{-}$and $v_{-}$ replaced by the local superpotential $W$ and the nonlocal superpotential $w$, respectively. Then we see that the potentials are written in terms of the superpotentials $W(x)$ and $w(x, y)$ as

$$
\begin{aligned}
V_{ \pm}(x)= & {[W(x)]^{2} \pm \frac{\hbar}{\sqrt{2 m}} \frac{d W(x)}{d x} } \\
v_{ \pm}(x, y)= & \int_{-\infty}^{\infty} d u w(x, u) w(u, y)+\{W(x)+W(y)\} w(x, y) \\
& \pm \frac{\hbar}{\sqrt{2 m}}\left[\frac{\partial w(x, y)}{\partial x}+\frac{\partial w(x, y)}{\partial y}\right] .
\end{aligned}
$$

If $w(x, y)$ is symmetric, then so are $v_{ \pm}(x, y)$. If $W(x)$ and $w(x, y)$ are real, so are $V_{ \pm}(x)$ and $v_{ \pm}(x, y)$.

Whereas in local models the relationship between the ground state and the superpotential $W(x)$ is one-to-one, this is not true in nonlocal models. Given the superpotentials $W(x)$ and $w(x, y)$ the zero-energy ground state $\psi_{0}(x)$ of $\hat{H}_{-}$is obtained from the integro-differential equation

$$
\frac{\hbar}{\sqrt{2 m}} \frac{d \psi_{0}(x)}{d x}+W(x) \psi_{0}(x)+\int_{-\infty}^{\infty} d y w(x, y) \psi_{0}(y)=0 .
$$

Conversely, for any normalized function $\psi_{0}(x)$, we could choose

$$
\begin{aligned}
w(x, y)=- & \frac{\hbar}{\sqrt{2 m}}\left[\psi_{0}^{\prime}(x) \psi_{0}(y)+\psi_{0}(x) \psi_{0}^{\prime}(y)\right] \\
& -\psi_{0}(x)\{W(x)+W(y)\} \psi_{0}(y) \\
& +C(x, y)
\end{aligned}
$$

Then $\psi_{0}(x)$ is the zero-energy ground state as long as arbitrary real functions $W(x)$ and $C(x, y)$ [ $=C(y, x)]$ satisfy the requirements

$$
\begin{gathered}
\int_{-\infty}^{\infty} d y W(y)\left[\psi_{0}(y)\right]^{2}=0, \\
\int_{-\infty}^{\infty} d y C(x, y) \psi_{0}(y)=0 .
\end{gathered}
$$

In order to determine higher energy eigenvalues exactly, we need to specify the superpotentials.

\section{A NONLOCAL DEFORMATION OF LOCAL MODELS}

As a specific example of the above formalism, we construct a class of exactly solvable models with both local and nonlocal potentials starting from any exactly solvable local model with a superpotential $W_{0}(x)$ which is an odd function of $x$. Examples are the harmonic oscillator with $W_{0}(x)=\sqrt{\frac{m}{2}} \omega x$ and the Rosen-Morse II or Scarf II potential with $W_{0}(x)=a \tanh \alpha x$. Now we choose

$$
\begin{aligned}
W(x) & =(1-c) W_{0}(x), \\
w(x, y) & =-c \frac{\hbar}{\sqrt{2 m}} \delta^{\prime}(x+y),
\end{aligned}
$$


where $c$ is a real parameter of nonlocality. Using Eq. (10) we obtain that

$$
\begin{gathered}
V_{ \pm}(x)=(1-c)^{2}\left[W_{0}(x)\right]^{2} \pm(1-c) \frac{\hbar}{\sqrt{2 m}} W_{0}^{\prime}(x) \\
\int_{-\infty}^{\infty} d y v_{ \pm}(x, y) \psi_{ \pm}(y) \\
=c^{2} \frac{\hbar^{2}}{2 m} \psi_{ \pm}^{\prime \prime}(x) \mp 2 c \frac{\hbar^{2}}{2 m} \psi_{ \pm}^{\prime \prime}(-x) \\
+c(1-c) \frac{\hbar}{\sqrt{2 m}} W_{0}^{\prime}(x) \psi_{ \pm}(-x)
\end{gathered}
$$

The nonlocal potential may be written as

$$
\hat{v}_{ \pm}=c^{2} \frac{\hat{p}^{2}}{2 m} \pm 2 c \frac{\hat{p}^{2}}{2 m} \hat{P}+c(1-c) \frac{\hbar}{\sqrt{2 m}} W_{0}^{\prime}(\hat{x}) \hat{P}
$$

where $\hat{P}$ is the parity operator. Thus the Hamiltonians $\hat{H}_{s}$ with $s= \pm$ are written as

$$
\begin{aligned}
\hat{H}_{s}=(1+ & \left.s \hat{P}_{c}\right)^{2} \frac{\hat{p}^{2}}{2 m}+(1-c)^{2}\left[W_{0}(\hat{x})\right]^{2} \\
& +s(1-c) \frac{\hbar}{\sqrt{2 m}} W_{0}^{\prime}(\hat{x})(1+s \hat{P} c) .
\end{aligned}
$$

We may mention that there is no classical analog of the present model since $\hat{H}_{ \pm}$contains the parity operator.

Since $\left[\hat{H}_{ \pm}, \hat{P}\right]=0$, we look for simultaneous eigenstates $\psi_{ \pm}(x)$ of $\hat{H}_{ \pm}$and $\hat{P}$. If $\hat{P} \psi_{s}(x)=\psi_{s}(-x)=P \psi_{s}(x)$ with $P= \pm 1$, then the eigenvalue equation for $\hat{H}_{s}$ becomes

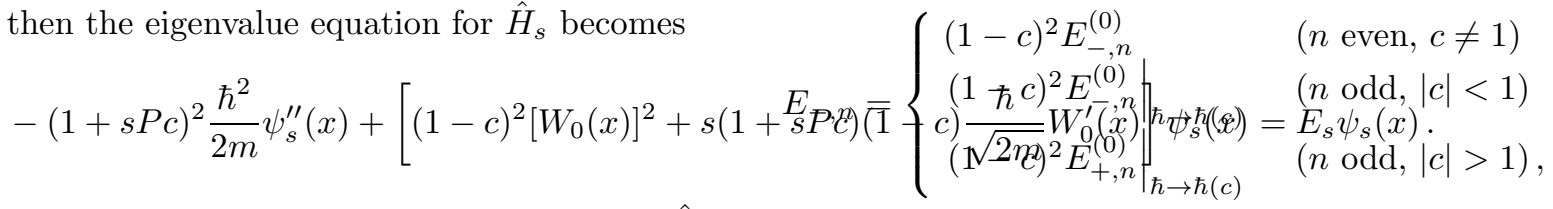

There are two singular cases $(c= \pm 1)$. At $c=1, \hat{H}_{ \pm}$ in the $P= \pm 1$ sector is a free Hamiltonian, which has no bound state. At $c= \pm 1, \hat{H}_{-}\left(\hat{H}_{+}\right)$has no kinetic term in the $P= \pm 1(P=\mp 1)$ sector. In the following we will disregard these cases.

We may represent $\hat{H}_{s}$ in terms of the local Hamiltonian $\hat{H}_{s}^{(0)}\left(=\hat{H}_{s}\right.$ with $\left.c=0\right)$ as follows: 19]

$$
\hat{H}_{s}= \begin{cases}(1-c)^{2} \hat{H}_{s}^{(0)} & (P=-s) \\ \left.(1-c)^{2} \hat{H}_{\alpha s}^{(0)}\right|_{\hbar \rightarrow \hbar(c)} & (P=+s),\end{cases}
$$

where the symbols $\alpha=\operatorname{sgn}[(1+c) /(1-c)]$ and $\hbar(c)=$ $\hbar|(1+c) /(1-c)|$ are introduced. We have $\alpha=+1$ for $|c|<1$ and $\alpha=-1$ for $|c|>1$. Note that the local Hamiltonians $\hat{H}_{s}^{(0)}$ are even in parity. The above relation (19) may be summarized in Table 1 . From the table or Eq. (19), we see, in particular, that for any $|c|>1$ $(\alpha=-1)$, both $\hat{H}_{+}$and $\hat{H}_{-}$in the given parity sector $P$ are proportional to $\hat{H}_{-P}^{(0)}$ (up to an appropriate rescaling of $\hbar$, when necessary), and thus both operators have normalizable zero-energy ground states.
We assume $\operatorname{sgn} W_{0}( \pm \infty)= \pm 1$, which ensures the existence of unbroken SUSY for local models $(c=0)$ where $\hat{H}_{-}^{(0)}$ has a zero-energy ground state. Then the normalized eigenstates $\psi_{s, n}^{(0)}(x)$ of $\hat{H}_{s}^{(0)}$ have energies $E_{s, n}^{(0)}$, where the nonnegative integer $n$ labels the number of nodes: $E_{-, 0}^{(0)}=0, E_{-, n}^{(0)}=E_{+, n-1}^{(0)}$, and $P=(-1)^{n}$. The eigenstates of $\hat{H}_{-}^{(0)}$ and $\hat{H}_{+}^{(0)}$ are related by

$$
\begin{aligned}
& \hat{A}^{(0)} \psi_{-, n}^{(0)}=\sqrt{E_{-, n}^{(0)}} \psi_{+, n-1}^{(0)}, \\
& \hat{A}^{(0) \dagger} \psi_{+, n}^{(0)}=\sqrt{E_{+, n}^{(0)}} \psi_{-, n+1}^{(0)},
\end{aligned}
$$

where $\hat{A}^{(0)}$ and $\hat{A}^{(0) \dagger}$ are given by Eq. (9) with $\hat{W}=\hat{W}_{0}$ and $\hat{w}=0$. Now we see that the normalized eigenfunctions of $\hat{H}_{ \pm}$are given by

$$
\begin{array}{r}
\psi_{-, n}(x)= \begin{cases}\psi_{-, n}^{(0)}(x) & (n \text { even, } c \neq 1) \\
\left.\psi_{-, n}^{(0)}(x)\right|_{\hbar \rightarrow \hbar(c)} & (n \text { odd },|c|<1) \\
\left.\psi_{+, n}^{(0)}(x)\right|_{\hbar \rightarrow \hbar(c)} & (n \text { odd },|c|>1),\end{cases} \\
\psi_{+, n}(x)= \begin{cases}\psi_{+, n}^{(0)}(x) & (n \text { odd }, c \neq 1) \\
\left.\psi_{+, n}^{(0)}(x)\right|_{\hbar \rightarrow \hbar(c)} & (n \text { even, }|c|<1) \\
\left.\psi_{-, n}^{(0)}(x)\right|_{\hbar \rightarrow \hbar(c)} & (n \text { even, }|c|>1) .\end{cases}
\end{array}
$$

The corresponding energy eigenvalues are given by

$$
E_{+, n}= \begin{cases}(1-c)^{2} E_{+, n}^{(0)} & (n \text { odd }, c \neq 1) \\ \left.(1-c)^{2} E_{+, n}^{(0)}\right|_{\hbar \rightarrow \hbar(c)} & (n \text { even, }|c|<1) \\ \left.(1-c)^{2} E_{-, n}^{(0)}\right|_{\hbar \rightarrow \hbar(c)} & (n \text { even, }|c|>1) .\end{cases}
$$

As in local models we have labelled the eigenfunctions by the number of their nodes. But in the nonlocal models, the energy eigenvalues are not ordered by the number of nodes. The operator $\hat{A}\left(\hat{A}^{\dagger}\right)$ converts an eigenfunction of $\hat{H}_{-}\left(\hat{H}_{+}\right)$into an eigenfunction of $\hat{H}_{+}\left(\hat{H}_{-}\right)$with the same energy. Explicitly,

$$
\begin{array}{r}
\hat{A} \psi_{-, n}(x)= \begin{cases}A_{-, n} \psi_{+, n-1}(x) & (n \text { even, } c \neq 1) \\
A_{-, n} \psi_{+, n-1}(x) & (n \text { odd },|c|<1) \\
A_{-, n} \psi_{+, n+1}(x) & (n \text { odd },|c|>1),\end{cases} \\
\hat{A}^{\dagger} \psi_{+, n}(x)= \begin{cases}A_{+, n} \psi_{-, n+1}(x) & (n \text { odd }, c \neq 1) \\
A_{+, n} \psi_{-, n+1}(x) & (n \text { even, }|c|<1) \\
A_{+, n} \psi_{-, n-1}(x) & (n \text { even, }|c|>1) .\end{cases}
\end{array}
$$

where $A_{s, n}=\operatorname{sgn}(1-c) \sqrt{E_{s, n}}$. We see that $\hat{A}$, when applied to eigenfunctions of $\hat{H}_{-}$, destroys a node in the 
even eigenfunction for $c \neq 1$ or in the odd eigenfunction for $|c|<1$, but creates an extra node in the odd eigenfuction for $|c|>1$. Similar behavior holds for the operator $\hat{A}^{\dagger}$. If $|c|<1$, then the ground state $\psi_{+, 0}(x)$ of $\hat{H}_{+}$has a positive energy and the SUSY is unbroken (Witten index $\Delta=1-0=1$ ) [6]. Howerver, if $|c|>1$, then the ground states $\psi_{-, 0}(x)$ and $\psi_{+, 0}(x)$ both have zero energy as discussed above, and are annihilated by $\hat{A}$ and by $\hat{A}^{\dagger}$, respectively. The SUSY is still unbroken for $|c|>1$ even though the Witten index $\Delta=1-1=0$. This case is similar to that with periodic potentials considered by Dunne et al. [16] who found that it is possible for both SUSY partners to have zero-energy ground states. Their ground states are not normalizable because they are Bloch states. However, normalizable are the eigenfunctions we obtained in the present paper.

It may be noted that at particular values of $c$ some of energy eigenvalues are doubly degenerate. In these cases the Hamiltonian $\hat{H}_{-}$(or $\hat{H}_{+}$) and the parity operator $\hat{P}$ form a complete set of commuting observables in contrast to the normal situation in one dimension, where the Hamiltonian alone is sufficient.

\section{CONCLUSIONS}

We have considered nonlocal potentials in connection with SUSY quantum mechanics. The local and nonlocal potentials are expressed in terms of the local and nonlocal superpotentials. We have presented a nonlocal extension of exactly solvable local models. Its energy eigenfunctions and eigenvalues are determined exactly and it is observed that both supersymmetric partners $\left(\hat{H}_{ \pm}\right)$may have, in some parameter range, normalizable zero-energy ground states. It would be interesting to find a (sufficient) condition of solvability generalizing the shape invariance which is applicable to models with both local and nonlocal potentials.

[1] A. L. Fetter and J. D. Walecka, Quantum Theory of Many-Paticle Systems (McGraw-Hill, 1971).

[2] H. A. Bethe and R. Jackiw, Intermediate Quantum Mechanics, Third Ed. (Benjamin/Cummings, 1986)

[3] J. M. Ziman, Principles of the Theory of Solids (Cambridge U. P., Cambridge, Eng., 1964).

[4] A. B. Balantekin, J. F. Beacom, and M. A. Candido Ribeiro, J. Phys. G 24, 2087 (1998).

[5] B. Nienhuis, Int. J. Mod. Phys. B 4, 929 (1990).

[6] E. Witten, Nucl. Phys. B 185, 513 (1981); Nucl. Phys. B 202, 253 (1982).

[7] For a recent review, see F. Cooper, A. Khare, and U. Sukhatme, Phys. Rep. 251, 267-385 (1995).
[8] F. Cooper, J. N. Ginocchio, and A. Khare, Phys. Rev. D 36, 2458-2473 (1987).

[9] L. É. Gendenshteîn, Pis'ma Zh. Eksp. Teor. Fiz. 38, 299302 (1983) [JETP Lett. 38, 356-359 (1984)].

[10] W. Kwong and J. L. Rosner, Prog. Theor. Phys. Suppl. 86, 366-376 (1986).

[11] R. Dutt, A. Khare, and U. Sukhatme, Phys. Lett. B 181, 295-298 (1986).

[12] J.-Y. Choi, J. Phys. A 26, L327-331 (1993).

[13] A. Das and S. A. Pernice, Mod. Phys. Lett. A 12, 581-588 (1997).

[14] P. K. Ghosh, A. Khare, and M. Sivakumar, Phys. Rev. A 58, 821-825 (1998).

[15] V. G. Bagrov and B. F. Samsonov, in the 7th Lomonosov conference proceeding 'Problems of Fundamental Physics' (Moscow, 1997)

[16] G. Dunne and J. Feinberg, Phys. Rev. D 57, 1271-1276 (1998); G. Dunne and J. Mannix, Phys. Lett. B 428, 115119 (1998).

[17] M. L. Glasser, Surf. Sci. 64, 141-156 (1977).

[18] In the position representation it may also be thought of as higher derivative terms $v\left(\frac{\hbar}{i} \frac{d}{d x}\right)$. The invariance under time reversal and the conservation of the probability require that $v(\hat{p})$ must be Hermitian and even in $\hat{p}$.

[19] Here it is assumed that $W_{0}(x)$ is independent of $\hbar$.

TABLE I. The representation of the Hamiltonians $\hat{H}_{s}$ in the parity sector $P= \pm 1$ in terms of the local Hamiltonians $\hat{H}_{s}^{(0)}\left(=\hat{H}_{s}\right.$ with $\left.c=0\right)$. The symbols $\alpha$ and $\hbar(c)$ are defined in the text.

\begin{tabular}{|c|c|c|}
\hline & $P=+1$ & $P=-1$ \\
\hline$\hat{H}_{-}$ & $(1-c)^{2} \hat{H}_{-}^{(0)}$ & $\left.(1-c)^{2} \hat{H}_{-\alpha}^{(0)}\right|_{\hbar \rightarrow \hbar(c)}$ \\
\hline$\overline{\hat{H}_{+}}$ & $\left.(1-c)^{2} \hat{H}_{\alpha}^{(0)}\right|_{\hbar \rightarrow \hbar(c)}$ & $\overline{(1-c)^{2} \hat{H}_{+}^{(0)}}$ \\
\hline
\end{tabular}

